\title{
A study on selection of probability distributions for at-site flood frequency analysis in Australia
}

\begin{abstract}
The most direct method of design flood estimation is at-site flood frequency analysis, which relies on a relatively long period of recorded streamflow data at a given site. Selection of an appropriate probability distribution and associated parameter estimation procedure is of prime importance in at-site flood frequency analysis. The choice of the probability distribution for a given application is generally made arbitrarily as there is no sound physical basis to justify the selection. In this study, an attempt is made to investigate the suitability of as many as fifteen different probability distributions and three parameter estimation methods based on a large Australian annual maximum flood data set. A total of four goodness-of-fit tests are adopted, i.e., the Akaike information criterion, the Bayesian information criterion, AndersonDarling test, and Kolmogorov-Smirnov test, to identify the best-fit probability distributions. Furthermore, the L-moments ratio diagram is used to make a visual assessment of the alternative distributions. It has been found that a single distribution cannot be specified as the best-fit distribution for all the Australian states as it was recommended in the Australian rainfall and runoff 1987. The log-Pearson 3, generalized extreme value, and generalized Pareto distributions have been identified as the top three best-fit distributions. It is thus recommended that these three distributions should be compared as a minimum in practical applications when making the final selection of the best-fit probability distribution in a given application in Australia.
\end{abstract}

Keyword: Floods; Flood frequency analysis; LP3 distribution; GEV distribution; Bayesian method; Rainfalls 